Original paper

\title{
A systematically compiled set of quantitative metrics to describe spatial characteristics of radiotherapy dose distributions and aid in treatment planning
}

\author{
Laura Patricia Kaplan $^{\text {a,b,c, }, \text {, Stine Sofia Korreman }}{ }^{\text {a,b,c }}$ \\ ${ }^{a}$ Department of Oncology, Aarhus University Hospital, Aarhus, Denmark \\ ${ }^{\mathrm{b}}$ Department of Clinical Medicine, Aarhus University, Aarhus, Denmark \\ ${ }^{\mathrm{c}}$ Danish Centre for Particle Therapy, Aarhus University Hospital, Aarhus, Denmark
}

\section{A R T I C L E I N F O}

\section{Keywords:}

Plan quality metrics

Treatment planning

Scripting

Spatial dose distribution

\begin{abstract}
A B S T R A C T
Purpose: Many quantitative metrics have been proposed in literature for characterization of spatial dose properties. The aim of this study is to work towards much-needed consensus in the radiotherapy community on which of these metrics to use. We do this by comparing characteristics of the metrics and providing a systematically selected set of metrics to comprehensively quantify properties of the spatial dose distribution.

Methods: We searched the literature for metrics to quantitatively evaluate dose conformity, homogeneity, gradient (overall and directional), and distribution and location of over- and under-dosed sub-volumes. For each spatial dose property, we compared the responses of its corresponding metrics to simulated dose variations in a virtual water phantom. Selection criteria were a metric's ability to describe simulated scenarios robustly and to be visualized in an intuitive way.

Results: We saw substantial differences in the responses of metrics to the simulated dose variations. Some conformity and homogeneity metrics were unable to quantify certain types of changes (e.g. target under-coverage). Others showed a large dependency on the shape and volume of targets and isodoses. Metric values differed between calculations in a static plan and in simulated full treatment courses including setup errors, especially for metrics quantifying distribution and location of hot and cold spots. We provide an Eclipse plugin script to calculate and visualize selected metrics.

Conclusion: The selected set of metrics provides complementary and comprehensive quantitative information about the spatial dose distribution. This work serves as a step towards broader consensus on the use of spatial dose metrics.
\end{abstract}

\section{Introduction}

The need for consistent treatment plan comparison is ubiquitous in clinical radiotherapy practice. Radiotherapy physicians and physicists often find themselves in a situation where they must select the optimal treatment plan for a patient among a group of competing plans. These plans might differ in modality (e.g. conformal vs. modulated treatments), planning technique (e.g. automated vs. manual), or simply be sequential iterations in the planning process. The status quo in treatment plan comparison is mainly built upon dose-volume histogram (DVH) metrics, visual inspection of dose distributions, and (to some extent) modeling of tumor control and normal tissue complication probabilities (TCP/NTCP).
The DVH is widely used as the main method of quantifying treatment plan quality, as many outcome models are built upon DVH parameters [1]. Most commercial treatment planning systems rely almost exclusively on DVH metrics for both plan optimization and evaluation routines. An important shortcoming of the DVH, however, is that information about the spatial distribution of dose within a target or organ-at-risk (OAR) is lost.

The spatial distribution of dose within a patient is mainly evaluated by visual inspection. However, it is near impossible for a human observer to gain a complete picture of the dose distribution's full threedimensional complexity from two-dimensional image slices. This makes visual inspection a lengthy and difficult task prone to observer biases and inter-observer variations [2-4]. Metrics that quantify spatial

\footnotetext{
* Corresponding author at: Department of Oncology, Aarhus University Hospital, Aarhus, Denmark.

E-mail address: lpkaplan@oncology.au.dk (L.P. Kaplan).
} 
characteristics of the dose distribution can help to minimize this subjectivity in plan comparison.

A vast number of such quantitative spatial dose metrics have been proposed in the literature. Approaches include indices to describe dose conformity (CI), homogeneity (HI), and gradients (GI) [5-27], defining and evaluating sub-volumes of anatomical structures [28,29], quantifying distances between volumes of interest [30-32], and using texture analysis methods to describe the dose distribution [29,33-39]. For many properties of interest, multiple different mathematical definitions for metrics have been put forth. A comprehensive, systematical comparison of the various metrics is lacking. Hence there is little foundation for choosing between metrics and arriving at a consensus on which to use $[40,41]$.

We aim to support the creation and selection of high-quality treatment plans by establishing a comprehensive toolbox of quantitative metrics to describe spatial dose distributions. To this end we have created an overview of spatial dose metrics described in the literature and selected a set of metrics which we assessed to be most suitable as an addition to the standard DVH metrics in use today. To perform this selection, we systematically tested and compared responses of all suggested metrics in simulated representative scenarios of several clinically relevant spatial dose distribution properties. Selection criteria included sensitivity to simulated dose variations as well as straightforward interpretability of the metric. Over a fractionated treatment course, the delivered dose is different from the static planned dose distribution. We investigated the effect this has on the metrics in scenarios with simulated random setup errors. Finally, we implemented calculation and visualization of the chosen set of metrics in a plugin script that can be run directly in the Eclipse treatment planning system as part of the treatment planning workflow.

In this paper, we focus on describing the different types of metrics and the process of selection. For any given treatment site and type, a selected subset of the metrics we describe here will likely be of clinical interest. Discussing these treatment-dependent aspects is outside the scope of this paper. Rather, our goal was to compile a comprehensive set of metrics from which those relevant to any given treatment context can be chosen. Selected clinical examples of the use of these metrics will be explored in future work.

\section{Methods}

This work consists of two main parts. The first is the quantitative comparison of published metrics for spatial dose characteristics. The second concerns the visualization of these metrics for use in a clinical plan comparison situation.

In this section we first introduce the metrics we have investigated. Following this, we describe the method of testing their response to simulated dose variations (both in static scenarios and including random inter-fraction shifts) and present the visualizations we considered to be intuitive and useful for quantitative plan comparison. Finally, we describe the methods used in developing an Eclipse plugin script to calculate and visualize the selected metrics.

\subsection{Quantitative metrics}

We identified spatial dose characteristics that might reasonably be factors in a plan's overall quality from international guidelines and recommendations (ICRU and RTOG), through literature review, and by directed questioning of experienced clinicians (two oncologists and five medical physicists; which metric categories were discussed with whom differed depending on each clinician's sub-specialty). These characteristic are: dose conformity, target dose homogeneity, dose gradient (overall and directional), and distribution/location of over-dosed (hot) or under-dosed (cold) volumes. Metrics that quantify these characteristics are described in detail in the following sub-sections 2.1.1-2.1.3.

Through extensive systematic searches on PubMed and Google
Scholar we identified reports of metrics to quantify these properties. Search words were combinations of "radiotherapy" or "radiation therapy" with one or several of the following: "plan quality", "conformity", "conformality", "homogeneity", "gradient", "dose fall-off", "index", "metric", "dose distribution", "spatial". For the dose gradient in a specific direction we could not find a previously published metric that contained the desired information. We therefore suggest new metrics to quantify this property (section 2.1.2).

In the following, we cover the various types of metrics and describe each investigated metric in detail.

\subsubsection{Indices to quantify overall dose conformity, homogeneity, and gradient}

We found a number of CIs, HIs, and GIs in the literature. Those we included in our analysis are listed in Table 1.

The CIs all in some way quantify the relationship between the target and prescription isodose volume, either in terms of these structures' volumes or their mean distance from each other. The HIs use the relationships between certain target DVH points (near-maximum and near-minimum) and/or the standard deviation of the differential target DVH. The GIs all in some way give the mean distance between two isodoses taken over all directions in space, but none contains any detailed information on the spatial distribution of dose within the patient.

Some of the indices in the literature combine a CI, HI, or GI with simple models for TCP or NTCP (e.g. Miften et al. [17]). These metrics were excluded from our analysis, as biological modeling was outside the scope of this work.

Some subgroups of metrics presented the same basic information but scaled it in different ways. For example, gradient indices proposed in $[9,20]$, and [23] are all based on the effective distance between two isodose volumes - but each index is formed by applying a different scaling to this distance. In cases like these, we included only one index, consisting simply of the dose parameters of interest without scaling, in our analysis.

\subsubsection{Directional quantification of dose gradient}

Cases exist in which the dose gradient is required to be steep in one particular direction (due to the target's proximity to an OAR for instance), while more shallow gradients are acceptable in other directions. In these cases, it can be useful to quantify the dose gradient in a specific anatomical direction. These metrics are distinct from those GIs that aim to quantify the overall effective dose gradient.

Our literature search found only one metric used to quantify the dose gradient in a specific direction: Gabryś et al. proposed an index giving the mean dose change in each cardinal direction over an OAR [34]. In its most general form, this metric can be defined as the change in dose between a point $r$ and a point moved in a direction $x$ by a distance vector $\boldsymbol{d}_{x}$ of length $d$ :

$G I_{d i r, x, d}=\operatorname{Dose}(\boldsymbol{r})-\operatorname{Dose}\left(\boldsymbol{r}+\boldsymbol{d}_{x}\right)$.

In clinical practice, however, it is often more interesting to consider the dose gradient in terms of specific isodoses. For this reason, we also propose another, complementary directional dose gradient metric which gives the distance between a reference volume (e.g. the target or the $100 \%$ isodose) and a queried isodose contour in a given direction. We investigated two variants that can be of clinical interest:

- Dose gradient along an anatomical coordinate system $\left(\mathrm{GI}_{\mathrm{dir}}\right)$ : The distance is defined along a line exiting the reference structure in the anterior, posterior, left, right, cranial, or caudal direction, or in principle any other direction $\times$ defined by superpositions of these base vectors:

$G I_{d i r, x, D}=\operatorname{Dist}_{x}\left(V_{\text {ref }}\right.$, Isodose $\left._{D}\right)$ 
Table 1

List of conformity, gradient, and homogeneity indices found in the literature.

\begin{tabular}{|c|c|c|}
\hline Index name & Definition & Reference and comments \\
\hline \multicolumn{3}{|l|}{ Conformity indices } \\
\hline Prescription Isodose Target Volume & $P I T V=\frac{V_{r e f}}{V_{T}}$ & [11] RTOG \\
\hline Conformity Index & $C I=\frac{V_{T, r e f}}{V_{r e f}}$ & [10] \\
\hline Conformity Number & $C N=\frac{V_{T, r e f}}{V_{T}} \times \frac{V_{T, r e f}}{V_{r e f}}$ & {$[7,14]$ ICRU Report 83} \\
\hline Geometric Conformity Index & $g C I=\frac{V_{T,<r e f}}{V_{T}}+\frac{V_{H, r e f}}{V_{T}}$ & {$[27]$} \\
\hline Dice-Sørensen Similarity Coefficient & $D S C=\frac{2\left|V_{T} \cap V_{\text {ref }}\right|}{\left|V_{T}\right|+\left|V_{r e f}\right|}$ & 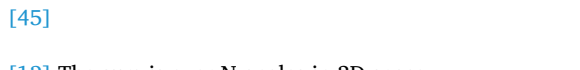 \\
\hline Conformity Index based on distance & $C I_{\text {distance }}=\frac{\sum_{i=1}^{N} \frac{d_{D}-d_{T}}{d_{T}}}{N} \times 100$ & [12] The sum is over $\mathrm{N}$ angles in 3D space \\
\hline Conformity Distance Index & $C D I=\frac{\left(V_{r e f}-V_{T, r e f}\right)+\left(V_{T}-V_{T, r e f}\right)}{1 / 2\left(S_{V r e f}+S_{\mathrm{TV}}\right)}$ & [13] \\
\hline \multicolumn{3}{|l|}{ Gradient indices } \\
\hline Gradient Index (volume ratio) & $G I_{\text {ratio, }, d}=\frac{V_{d}}{V_{r e f}}$ & {$[19,25] V_{d}=V_{50 \%}$ and $V_{r e f}=V_{P D}$ are commonly used } \\
\hline Gradient Index (effective isodose distance) & $G I_{\text {dist }, d}=\sqrt[3]{V_{d}}-\sqrt[3]{V_{r e f}}$ & {$[9,20,31]$ Different mathematical transformations applied } \\
\hline Gradient Curve Index & $G C I_{d}=\sum_{j=i}^{D_{\text {ref }}-d} \frac{V_{i}-V_{i+d}}{1 / 2\left(S_{i}+S_{i+d}\right)}$ & [22] Mean effective distance between isodose $d$ and $V_{r e f}$ \\
\hline \multicolumn{3}{|l|}{ Homogeneity indices } \\
\hline Homogeneity Index (ICRU) & $H I_{I C R U}=\frac{D_{2 \%}-D_{98 \%}}{D_{50 \%}}$ & [14] ICRU Report 83 \\
\hline Homogeneity Index (RTOG) & $H I_{R T O G}=\frac{D_{\max }}{D_{m}}$ & [11] RTOG \\
\hline Homogeneity Index (Mayo et al.) & $H I_{\text {Mayo et al. }}=\frac{D_{\max }}{D_{n}} \times \frac{D_{S T D}}{D_{n}}$ & [23] \\
\hline Homogeneity Index (Heufelder et al.) & $H I_{\text {Heufelder }}=e^{-\left[0.1 \times\left(1-\frac{D_{\text {mean }}}{D_{p}}\right)\right]^{2}} \times e^{-\left(0.1 \times D_{\text {STD }}\right)}$ & [16] \\
\hline Dose standard deviation & $D_{S T D}=\sqrt{\frac{1}{N} \sum_{i=1}^{N}\left(D_{i}-D_{\text {mean }}\right)}$ & $N$ : Number of voxels in structure \\
\hline
\end{tabular}

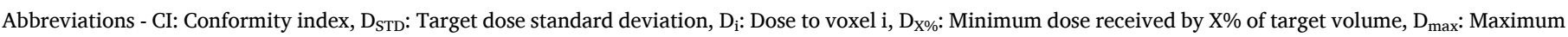

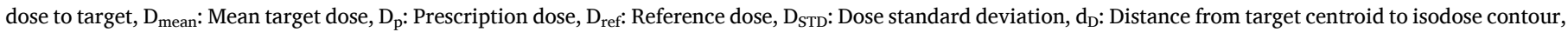

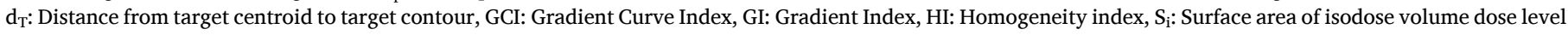

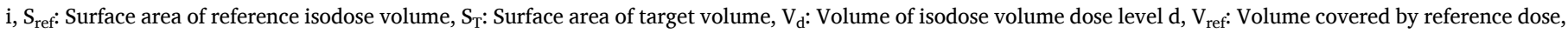

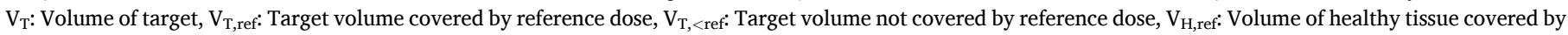
reference dose.

This method is illustrated in Fig. 1, panels A and B.

- Dose gradient towards an OAR ( $\left.\mathrm{GI}_{\mathrm{OAR}}\right)$ : The shortest distance between the OAR and the queried isodose. This can be given as either the absolute shortest distance between the two volumes or the mean distance between a certain percentile of points. For illustration purposes we show the mean distance for the 10-percentile of points in the OAR contour with the shortest distance to the isodose:

$$
G I_{O A R, D, 10 \%}=\frac{\sum_{i}^{N \times 0.1} \min _{j}\left(\operatorname{Dist}\left(\text { OAR }_{i}, \text { Isodose }_{D, j}\right)\right)}{N \times 0.1},
$$

where $I$ sodose $e_{D, j}$ are points $j$ in the isodose contour of dose $D$ and $N$ is the number of points $O A R_{i}$ in the OAR's contour which are ordered in ascending order by distance to the isodose. This is illustrated in Fig. 1, panels C and D.

These three variants (dose-at-distance, distance-to-dose, and doseoutside-OAR) contain complementary information about the dose distribution, and each one can be the most interesting depending on the clinical situation. The three directional metrics were compared to the general gradient index GCI in simulated scenarios to exemplify their usefulness.

\subsubsection{Distribution of under- and over-dosed volumes}

In fractionated treatments, large contiguous under-dosed volumes in the target (cold spots) are likely to be under-dosed in many consecutive treatment fractions and can thus be considered detrimental to overall plan quality. At the same time, the same absolute cold volume may be acceptable if it is distributed in multiple smaller spots, as these are likely to move slightly with respect to the patient anatomy for every fraction. Thus, they would not result in the same cells being under-dosed every time when accumulated over many fractions.

We found two metrics in the literature to quantify distribution of cold (or hot) volumes: the LCS (Large Cold Spot) and $\mathrm{LCS}_{\mathrm{D}}$ scores proposed by Said et al. [29]. These rely on a connected component labelling (CCL) algorithm [42] to identify contiguous sub-volumes within the target. Given the three-dimensional dose matrix, this algorithm identifies contiguous sub-volumes under or over a given cut-off dose value or within a given dose range. The LCS index is based on the full Grey Level Size Zone Matrix [43] of the target. To determine this matrix, a large number of CLL calculations is needed, which makes the LCS metric very computation intensive. The $\mathrm{LCS}_{\mathrm{D}}$ index uses a binary classification of target voxels as either under(over)-dosed or not, and thus requires only a single CCL calculation. Of these two very similar metrics, we included only the $\mathrm{LCS}_{\mathrm{D}}$ in our analysis. In addition to the $\mathrm{LCS}_{\mathrm{D}}$, we investigated a metric consisting simply of the number and respective size of the separate cold or hot sub-volumes. Since the $\mathrm{LCS}_{\mathrm{D}}$ itself is based on the size and number of cold spots, both metrics inherently contain the same information, and this was solely a comparison of which metric visualization was preferable.

\subsubsection{Location of under- and overdosed volumes}

The impact of a hot or cold volume on plan quality varies depending on its location within the patient anatomy. A cold spot close to the target's edge may be acceptable, for example, while the same cold spot located at the target's center would render the plan unacceptable. Analogously, hot areas in OARs located close to the target's edge might 

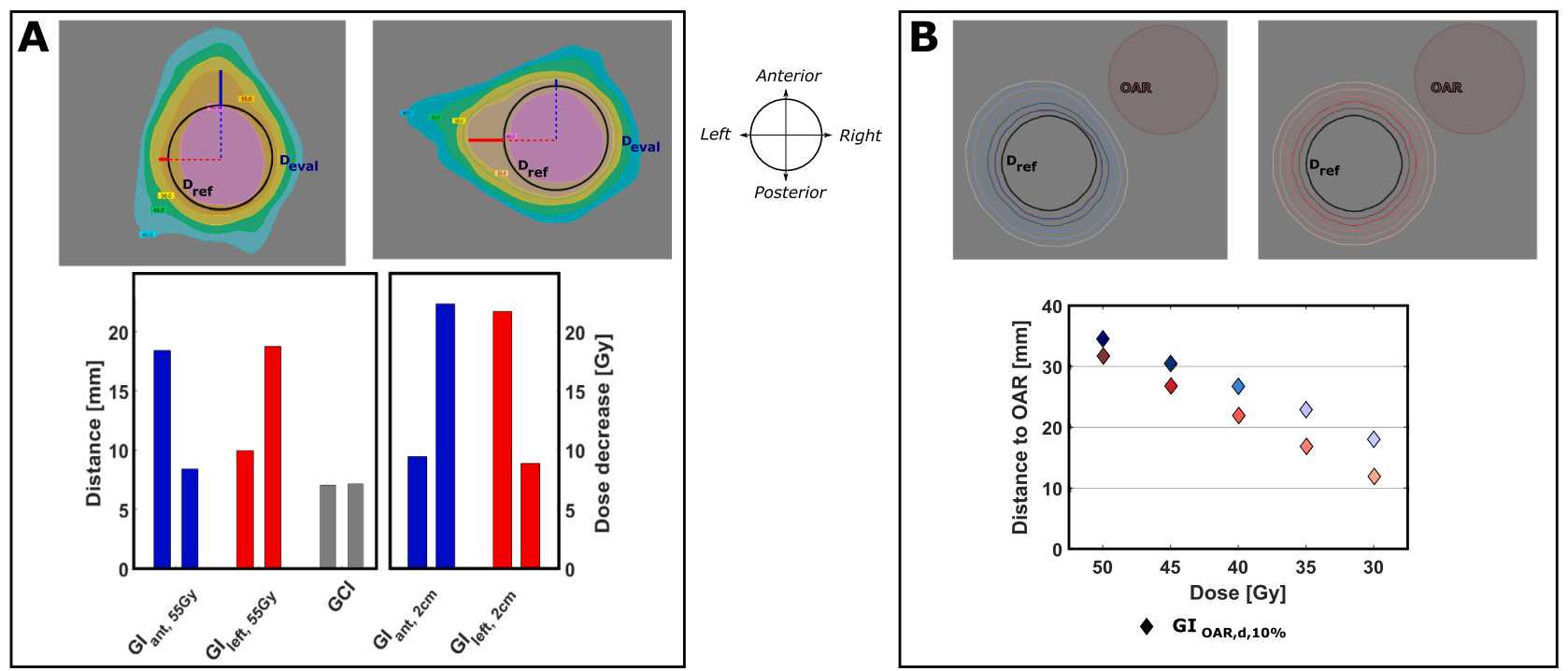

Fig. 1. Spatial quantification of the dose gradient. A: GI_\{ant, 55 Gy\} (blue, left plot), GI_\{ant, $2 \mathrm{~cm}\}$ (blue, right plot), GI_\{right, 2 cm\}(red, left plot), GI_\{right, 55 Gy\}(red, right plot), and GCI (grey, left plot) for two scenarios: a shallower gradient towards the anterior (upper row, left panel) and towards the left (uper row, right panel). Each pair of bars shows one metric calculated in each of the two depicted scenarios: the left bar corresponding to the anterior shallow gradient and the right bar to the left shallow gradient. B: GI_\{OAR, d, 10\%\} at several dose levels for two scenarios: a gradient that is more steep towards the OAR (upper row, left panel blue markers) and an isotropic gradient (upper row, right panel - red markers). (For interpretation of the references to colour in this figure legend, the reader is referred to the web version of this article.)

be unavoidable, while they should be avoided in areas far from any target. The most straightforward way to quantify dose location is by giving a voxel's distance to a reference point, line, or volume. Our investigation therefore focused on determining the optimal method to visualize this quantity.

The visualization methods we found in literature were the SpatialDVH as proposed by Zhao et al. [28], the dose-location histogram (DLH) as implemented in the Computational Environment for Radiology Research (CERR) [30], and the vectorized DVH (vDVH) proposed by Mayo et al. [31]. In addition, we developed a min/max voxel distance
(VDist $\left.\mathrm{min}_{\max }\right)$ as a more intuitive alternative to existing visualization methods. Here, the minimum distance of each voxel in the cold/hot volume to the reference volume's edge is found. The plot then shows the smallest and largest of these distances, as well as the 95 and 5 percentile distances, as exemplified in Fig. 1.

\subsection{Simulated treatment scenarios}

We simulated a set of idealized dose distribution scenarios to explore the performance of the metrics described above. These scenarios

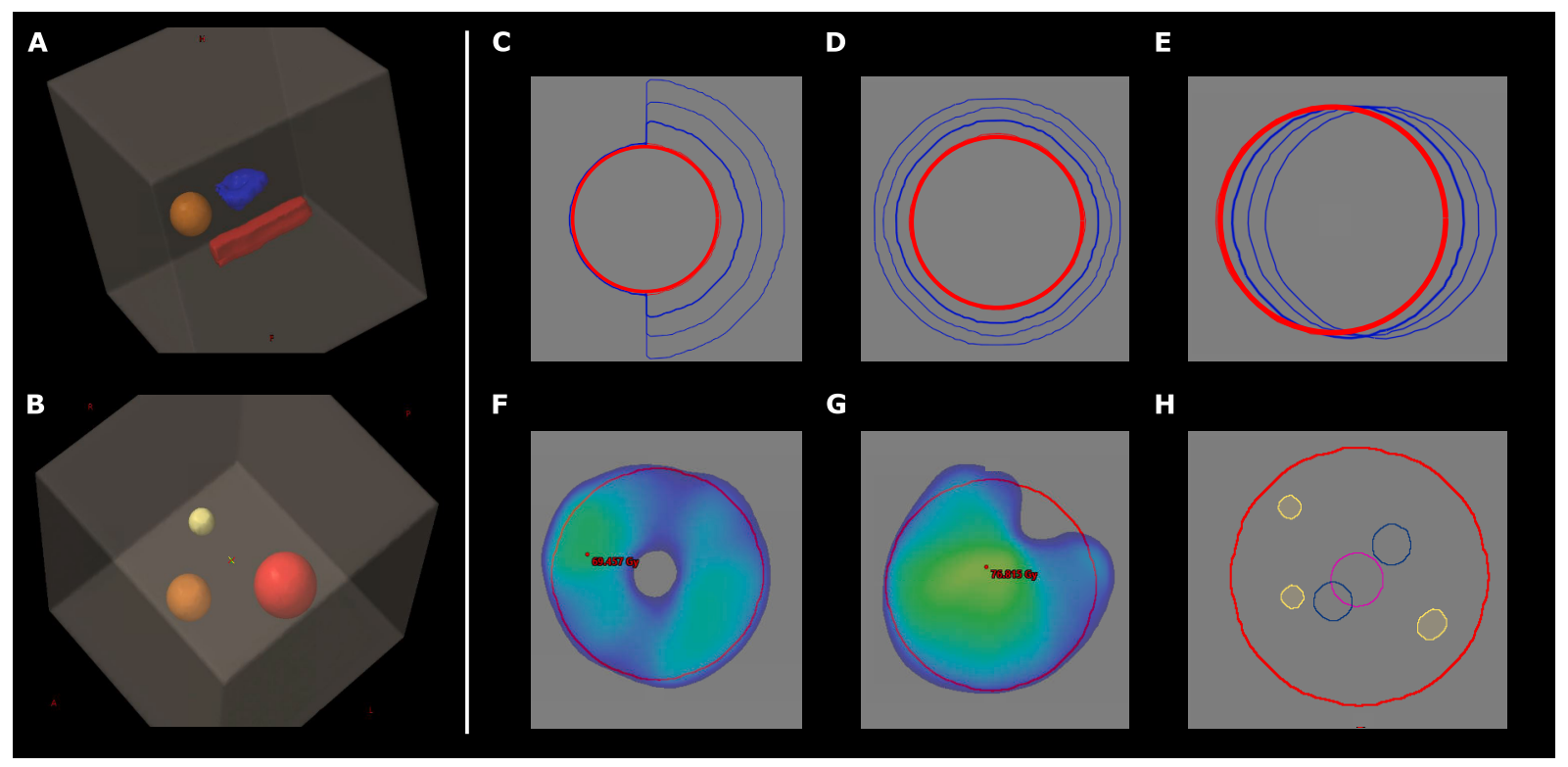

Fig. 2. Illustration of some of the simulated dose distribution variations. Panels A and B show simulated targets of differing shape (A) and volume (B). Panels C through H show simulated dose distribution scenarios. Upper row: Anisotropic (C) and isotropic (D) expansion as well as lateral shift (E) of the target (red). Prescription isodose volume shown in blue. Lower row: Simulated cold volumes centrally (F) and close to the target's edge (G) as well as a total cold volume of 1.56 cc in either one (pink), two (blue), or fourteen (yellow) contiguous sub-volumes ( $\mathrm{H}$, not all yellow cold spots are seen on the presented axial slice). (For interpretation of the references to colour in this figure legend, the reader is referred to the web version of this article.) 
represent variations of clinically relevant plan characteristics, ranging in degree from small to extreme, which the metrics should be able to describe quantitatively.

Target, OAR, and isodose structures were generated in a virtual $30 \times$ $30 \times 30 \mathrm{~cm}^{3}$ water phantom in the treatment planning software Eclipse v. 13.7 (Varian Medical Systems, Palo Alto, CA). We placed target structures of different shapes and sizes (see below) at the center of the phantom and a spherical OAR (diameter $8.3 \mathrm{~cm}$ ) at a distance (center of mass) of $10.6 \mathrm{~cm}$ from the largest spherical target. For conformity and gradient indices, we did not simulate a full dose matrix. Instead, isodose contours for evaluation of metrics were created by expanding, contracting, or geometrically shifting the contour of the target in question. Axial CT slices and 3D renderings of some scenarios are shown in Fig. 2. For dose distribution and location metrics, we simulated VMAT treatment plans. Optimization objectives for these are given in the Supplementary Material, Tables S1-S4. For homogeneity indices, we calculated DVHs for different dose probability density functions (corresponding to idealized differential DVHs). These are shown in Supplementary Figure S1.

All calculation of metrics was performed using the C\#-based Eclipse Scripting Application Programming Interface (ESAPI; Varian Medical Systems). Table 2 lists the simulated scenarios for each plan characteristic, and Fig. 2 panels $\mathrm{C}-\mathrm{H}$ show a selection of scenarios. This part of the analysis was binary: either a metric reacted to a change or it did not.

We further varied the target volume and shape independently to determine the dependency of conformity and gradient metrics on these factors. Investigated shapes were spherical, oblong, and irregular. To vary the volume, we simulated spheres with diameter 3,5 , and $7 \mathrm{~cm}$, corresponding to volumes of 13.6, 64.7, and $179.2 \mathrm{cc}$. See Fig. 2 panels A and B. The shape and volume dependence was then quantified as the difference between the lowest and highest value of these five data points (sphere $3 \mathrm{~cm}$, sphere $5 \mathrm{~cm}$, sphere $7 \mathrm{~cm}$, irregular, oblong) scaled to the lowest value:

variation $=\frac{\max _{\text {shape,volume }}(\text { metric })-\min _{\text {shape,volume }}(\text { metric })}{\min _{\text {shape,volume }}(\text { metric })}$

This was done for each metric using the first (leftmost) data point series in Fig. 3, panels A and D.

Criteria for selecting a comprehensive, complimentary set of metrics were both their ability to describe the simulated test scenarios as well as their potential to be presented to a plan evaluator in an intuitive and easy-to-understand manner. This last part was evaluated through targeted assessment discussions with the involved clinicians: Metric visualizations that required extensive time and explanation until clinicians felt they understood them were deemed non-intuitive.

\subsubsection{Robustness analysis}

The planned dose distribution does not equal the dose distribution that is actually delivered to a patient over multiple fractions. Each fraction has a probability of positioning errors that can influence the final delivered dose distribution. We investigated the influence of random errors on the metrics described above by simulating entire treatment courses. Systematic errors were not considered in this analysis, as their effect is only well described at the population level, and the metrics we investigate here are for use on a per-patient basis.

In this analysis a course was simulated to consist of 30 fractions. In each fraction, the dose matrix was rigidly moved in a random direction, given by a $3 \mathrm{D}$ vector with $\mathrm{x}, \mathrm{y}$, and $\mathrm{z}$ components randomly sampled from a normal distribution with mean $0 \mathrm{~mm}$ and standard deviation 3 $\mathrm{mm}$. We generated 50 such courses for each simulated dose variation scenario. For simulated dose scenarios where full dose matrices were not calculated, the binary structure mask matrix of the isodose was moved instead. Each voxel's total simulated dose was then proportional to the

Table 2

Simulated dose distribution scenarios for each characteristic of interest.

\begin{tabular}{|c|c|c|c|}
\hline & $\begin{array}{l}\text { Dose variation type } \\
\text { - Scenario }\end{array}$ & $\begin{array}{l}\text { Dose variation type } \\
\text { - Scenario }\end{array}$ & $\begin{array}{l}\text { Dose variation type } \\
\text { - Scenario }\end{array}$ \\
\hline Conformity & $\begin{array}{l}\text { PIV = PTV } \\
+ \text { outer margin: } \\
\text { - } 5 \mathrm{~mm} \\
\text { - } 10 \mathrm{~mm} \\
\text { - } 15 \mathrm{~mm} \\
\text { (Fig. 2, panel D) }\end{array}$ & $\begin{array}{l}\text { PIV }=\text { PTV } \\
\text { - inner margin: } \\
\text { - } 3 \mathrm{~mm} \\
\text { - } 4 \mathrm{~mm} \\
\text { - } 5 \mathrm{~mm}\end{array}$ & $\begin{array}{l}\text { PIV = PTV } \\
\text { shifted laterally: } \\
\text { - } 5 \mathrm{~mm} \\
\text { - } 10 \mathrm{~mm} \\
\text { - } 15 \mathrm{~mm} \\
\text { (Fig. 2, panel E) }\end{array}$ \\
\hline Homogeneity & $\begin{array}{l}\text { Constant dose distribution: } \\
P(d)= \begin{cases}k, & 95 \%<d<107 \% \\
0, & \text { else }\end{cases} \end{array}$ & $\begin{array}{l}\text { Gaussian dose distribution: } \\
\begin{array}{l}P(d)=\frac{1}{\sigma \sqrt{2 \pi}} e^{-\frac{(d-\mu)^{2}}{\sigma^{2}}} \\
\text { - } \mu=102 \%, \sigma=2 \% \\
\bullet \mu=102 \%, \sigma=3 \% \\
\text { - } \mu=104 \%, \sigma=2 \%\end{array}\end{array}$ & $\begin{array}{l}\text { Skew normal dose distribution: } \\
\begin{array}{l}P(d)=\frac{1}{\omega \sqrt{2 \pi}} e^{-\frac{((d-\mu) / \omega)^{2}}{2}}\left(1+\operatorname{Erf}\left(\frac{\alpha(d-\xi)}{\omega \sqrt{2}}\right)\right) \\
\bullet \xi=102, \omega=2, \alpha=+4 \\
\bullet \xi=102, \omega=2, \alpha=-4\end{array}\end{array}$ \\
\hline Gradient & $\begin{array}{l}\text { Isotropically decreasing gradient. } \\
\text { Distance } \mathrm{V}_{\mathrm{d}} \text { to } \mathrm{V}_{\text {ref: }} \text { : } \\
\text { - } 10 \mathrm{~mm} \\
\text { - } 20 \mathrm{~mm} \\
\text { - } 30 \mathrm{~mm}\end{array}$ & $\begin{array}{l}\text { Gradient decreasing along } 75 \% \text { of target's surface: } \\
\text { Distance } V_{\mathrm{d}} \text { to } \mathrm{V}_{\text {ref: }} \text { : } \\
\text { - } 10 \mathrm{~mm} \\
\text { - } 20 \mathrm{~mm} \\
\text { - } 30 \mathrm{~mm}\end{array}$ & $\begin{array}{l}\text { Gradient decreasing along } 50 \% \text { of target's surface: } \\
\text { DistanceV } V_{\mathrm{d}} \text { to } \mathrm{V}_{\text {ref: }} \\
\text { - } 10 \mathrm{~mm} \\
\text { - } 20 \mathrm{~mm} \\
\text { - } 30 \mathrm{~mm} \\
\text { (Fig. 2, panel C) }\end{array}$ \\
\hline Directional gradient & $\begin{array}{l}\text { Dose gradient shallower towards one direction: } \\
\text { - Anterior } \\
\text { - Right } \\
\text { - OAR center-of-mass }\end{array}$ & & \\
\hline Distribution & $\begin{array}{l}\text { Target cold volume }=1.56 \mathrm{~cm}^{3} \text { as: } \\
\text { - one spot } \\
\text { - two spots } \\
\text { - } 14 \text { spots } \\
\text { (Fig. 2, panel H) }\end{array}$ & & \\
\hline Location & $\begin{array}{l}\text { Target cold volume: } \\
\text { - At center } \\
\text { - At edge } \\
\text { (Fig. 2, panels F and G) }\end{array}$ & $\begin{array}{l}\text { OAR hot volume: } \\
\text { - Close to target } \\
\text { - Far from target }\end{array}$ & \\
\hline
\end{tabular}

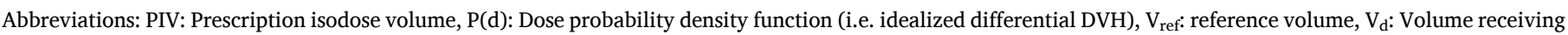
at least certain evaluation dose level. 


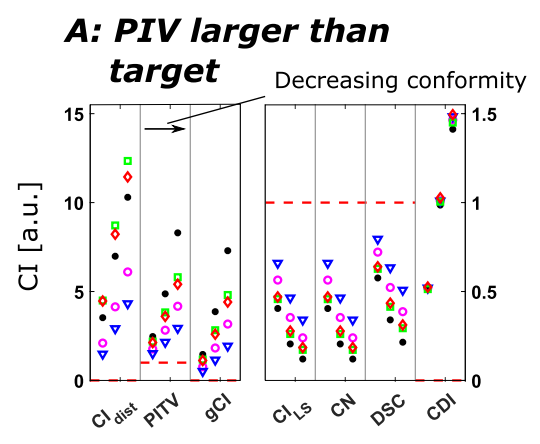

\section{D: Isotropic gradient}

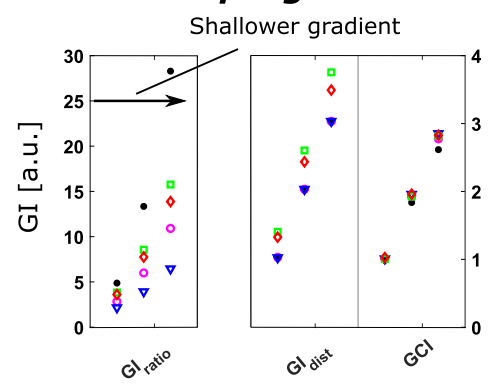

\section{B: PIV smaller than} target

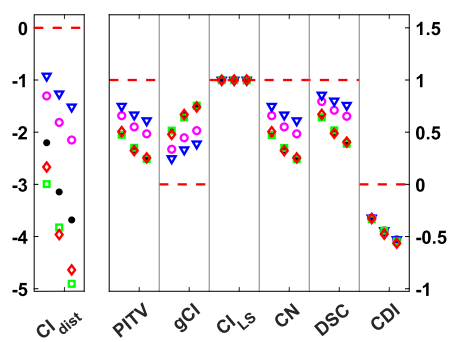

\section{C: PIV shifted laterally}

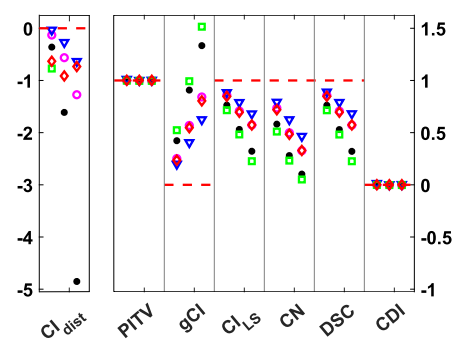

Target/Reference isodose shapes:

\section{E: Gradient along 75\% of target surface}
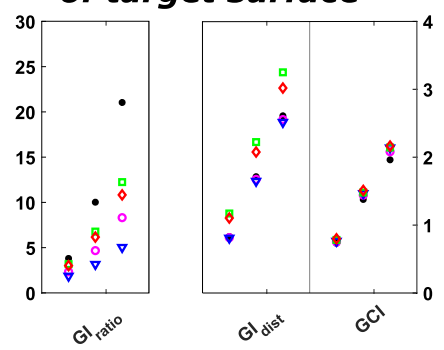

\section{F: Gradient along $50 \%$ of target surface}

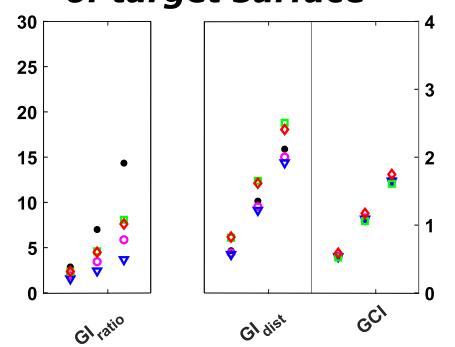

Sphere
$D=3 \mathrm{~cm}$$\quad \begin{gathered}\text { Sphere } \\ D=5 \mathrm{~cm}\end{gathered}$

Fig. 3. Sensitivity of conformity and gradient indices to simulated changes in the dose distribution, target shape, and target volume. Upper row (A-C): Conformity indices' values in simulated scenarios of increasingly compromised dose conformity to the target. Dose conformity decreases from left to right in a cell. Each cell shows values for one metric. These scenarios are described in Table 2, column 1. Scenarios corresponding to plots A and C are illustrated in Fig. 2, panels D and E, respectively. Ideal values for each index (i.e. coverage matching the target perfectly) are shown as red dashed lines. The value of $\mathrm{CI}_{\text {dist }}$ in the two last scenarios in plot $\mathrm{C}$ for the oblong target shape are extremely low ( -93.36 and -40.84 for $1 \mathrm{~cm}$ and $1.5 \mathrm{~cm}$ PIV shifts, respectively) and are not shown in the plot for this reason. Lower row (D-F): Gradient indices' values in simulated scenarios of increasingly shallow dose gradient. Dose gradient steepness decreases from left to right in a cell. Each cell shows values for one metric. These scenarios are described in Table 2, column 3. Scenarios corresponding to the plots to the left and right are illustrated in Fig. 2, panels D and C, respectively. PIV: Prescription isodose volume. (For interpretation of the references to colour in this figure legend, the reader is referred to the web version of this article.)

number of fractions in which it was covered by the isodose.

The robustness analysis was programmed in MATLAB, using functions from the CERR package [30].

\subsection{Plan evaluation plugin script}

The metrics were implemented in a binary plugin script using C\# and ESAPI. We created scripts for both Eclipse version 13.7 and 15.6 (Varian Medical Systems). The Windows Presentation Foundation framework was used to create a graphical user interface for the script, and the OxyPlot package for C\# (v. 1.0.0, https://oxyplot.github.io/) to visualize the metrics in plots of various types.

All metrics describing the distribution of sub-volumes were calculated using the same connected-component-labeling algorithm [42]. Dose matrices were sampled at a resolution of $1 \times 1 \times 1 \mathrm{~mm}^{3}$.

For the $G I_{d i r, x, d}$ as shown in Fig. 1, we calculated the distance between the reference structure and queried isodose in the anterior and left directions on each axial CT slice, along a line crossing the reference structure's center-of-mass left-right (LR) or anterior-posterior (AP) coordinate. To increase calculation robustness, we calculated the mean distance for three parallel lines spaced $1 \mathrm{~mm}$ apart in our implementation of this metric.

For fast calculation of the minimum distance between points in two contours (for the $G I_{O A R}$ and the $V D_{\min \text {,max }}$ ) we used a k-d tree algorithm [44].

\section{Results}

In the following section we describe the results obtained by calculating the various metrics in simulated dose distribution scenarios, first for the static scenarios and then considering simulated random geometrical errors. Based on these results, we then present the selected set of metrics and visualizations that constitutes our suggestion for a comprehensive toolbox for detailed, quantitative description of treatment plans. Finally, we present the script we developed to calculate these metrics from within the Eclipse treatment planning system.

\subsection{Metrics' performance in simulated static dose distribution scenarios}

All numerical results can be found in supplementary Tables S5-S12 and Figures S5-S8. In the following we summarize our main results. For conformity, homogeneity, and overall gradient indices, these are the quantitative results of calculating each metric in the simulated static dose scenarios. For the directional dose gradient, dose distribution, and dose location metrics, we also report the visualization most suited for intuitive quantitative plan comparison, based on targeted discussions with experienced clinicians.

\subsubsection{Conformity}

Fig. 3 (upper row) shows responses of all investigated conformity metrics to the simulated variations in dose conformity. All conformity metrics were sensitive to over-coverage of the target. The conformity 
number (CN, [7]), the Dice-Sørensen similarity coefficient (DSC, [45]), the geometric conformity index (gCI, [27]) and the distance conformity index $\left(\mathrm{CI}_{\text {dist }},[12]\right)$ could distinguish between all simulated scenarios. Of the investigated metrics, the conformity distance index (CDI, [13]) showed least response variation with changing target shape (0.02) and volume (0.01), while $\mathrm{CI}_{\text {dist }}$ had the highest variation with shape (1.14) and $\mathrm{gCI}$ with volume (0.65).

\subsubsection{Homogeneity}

The simulated scenarios were designed to show dose variations within $95 \%-107 \%$ of the prescribed dose, an interval which is commonly considered clinically acceptable [14](DVHs are shown in Figures S1 and S2, Supplementary material). The values of all investigated metrics in these scenarios are shown in Supplementary Table S8. Only the HI proposed in ICRU guidelines ( $\mathrm{HI}_{\mathrm{ICRU}}$, [14]) and the HI proposed by Mayo et al. [23] were able to distinguish between all simulated scenarios, though the numerical difference between values for the two skewed normal dose distributions (shown in Fig. S1, panels E and F) was small for both.

\subsubsection{Gradient}

Results for all investigated gradient metrics in all simulated scenarios are shown in Fig. 3 (lower row). All investigated gradient indices were able to distinguish between the different dose distributions. Of all these metrics, the distance-based gradient index $\left(\mathrm{GI}_{\mathrm{dist}}\right)$ varied least as a function of spherical target volume (0.00) and the Gradient Curve Index (GCI) [22] with varying shape (0.40). In addition, GCI values for the anisotropically expanded target approximately scaled with the percentage of the target's surface that was expanded.

\subsubsection{Directional quantification of dose gradient}

Plots A and B in the lower row of Fig. 1 show the $\mathrm{GI}_{\text {dir }}$ calculated for the two scenarios shown in panels A and B above each plot. The gradient in the anterior and left directions was found along a line going from the target's center-of-mass outwards in each of the two directions (as shown in the upper row of Fig, 1). Both the directional gradient variant defined by dose change at distance $d\left(G I_{d i r, x, d}\right)$ and the variant defined as distance to dose $D\left(G I_{d i r, x, D}\right)$ clearly discriminated between scenario A, where the evaluation isodose has a "bulge" in the anterior direction, and scenario $\mathrm{B}$, where the bulge is towards the left of the reference structure. The GCI could not discriminate between these two scenarios.

Plots $\mathrm{C}$ and D show the $\mathrm{GI}_{\mathrm{OAR}, \mathrm{d}, 10 \%}$ for the OAR structure and isodoses shown in panels $C$ and $\mathrm{D}$ above the plots. This metric was able to clearly discriminate between an isotropic dose gradient (panel D) and a gradient that is steeper towards the OAR (panel C).

\subsubsection{Distribution of under- and over-dosed volumes}

The connected component labeling algorithm correctly identified separate contiguous cold volumes. Both the metric consisting simply of number and size of each sub-volume and the $\mathrm{LCS}_{\mathrm{D}}$ score were thus able to discern between the three simulated scenarios.

Clinical experts evaluated both metrics and found that simply stating size and volume of each cold sub-volume yields the most descriptive and intuitive. The chosen visualization method is shown in Fig. 4, panel A.
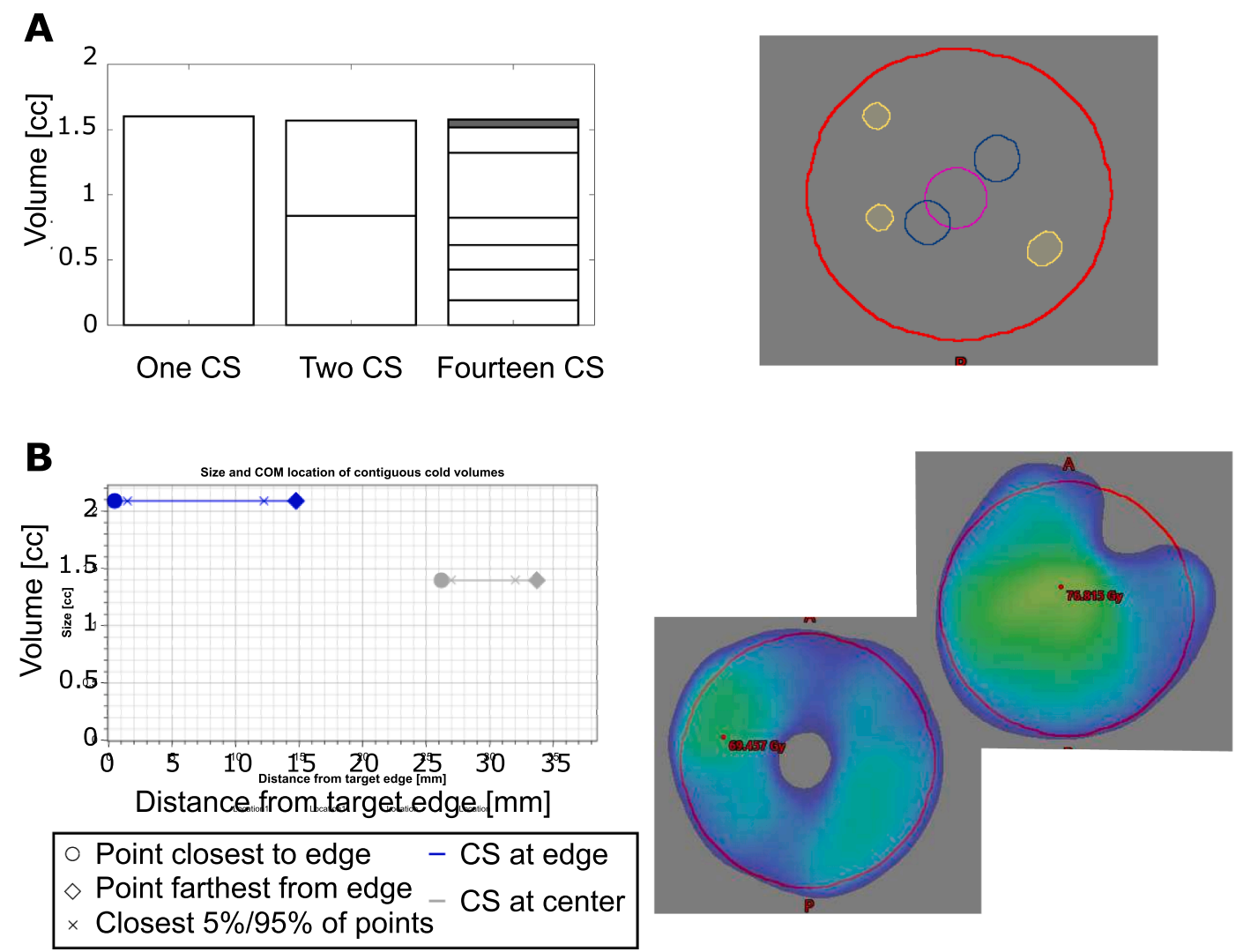

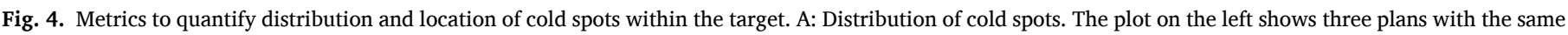

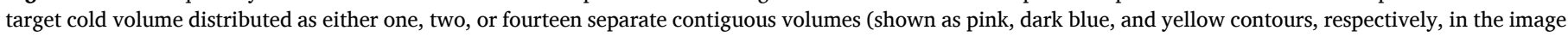

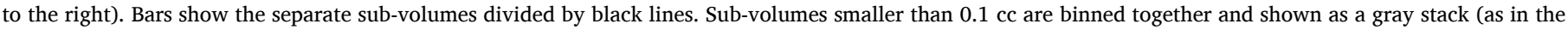

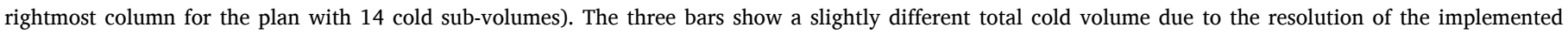

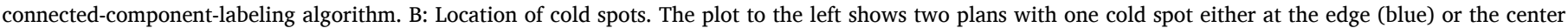

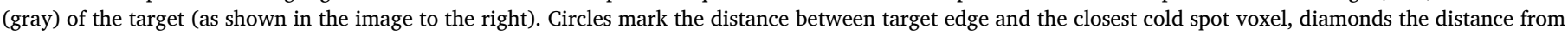

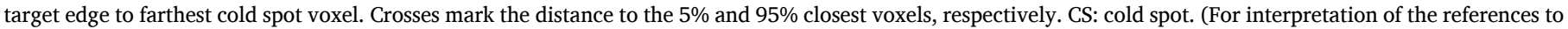
colour in this figure legend, the reader is referred to the web version of this article.) 


\subsubsection{Location of under- and over-dosed volumes}

All investigated metrics were able to discern between the simulated scenarios (see Figures S5-S8, Supplementary Material).

Regarding visualizations we found the following: The SpatialDVH was deemed intuitive in its presentation but differences between plans were not straightforward to quantify in a single plot. The DLH representation was deemed not intuitive enough, as it took even senior clinical physicists some time to familiarize themselves with it and interpret it. Clinicians found the VDist ${ }_{\min / \max }$ easy to interpret, while containing relevant information about the location of over- or under-dosed volumes. An example is shown in Fig. 4, panel B.

\subsection{Robustness analysis}

For all investigated metrics, the calculated values changed in simulations of a full treatment course with random setup errors compared to planned static scenarios. The degree of the difference depended strongly on the type of dose variation that was simulated. We highlight general findings below.

\subsubsection{Distribution metrics}

The simulated dose scenarios with varying numbers of cold spots (see Fig. 2, H) were quite sensitive to random positioning errors. This was reflected in the spread of both the number and volumes of contiguous cold spots and of the total cold volume in the simulated fractionated treatments (Fig. 5, A). In general, the scenario with the total cold volume split into fourteen separate spots (in the planned dose) tended to result in smaller total cold volumes and fewer contiguous spots over $0.1 \mathrm{cc}$ in fractionated scenarios.

\subsubsection{Location metrics}

In the simulated scenarios of over- or under-dosed volumes in different locations, the blurring of the dose in fractionated treatment courses also led to smaller cold and hot spots than in the planned nominal dose. The VDist ${ }_{\min / \max }$ clearly showed this volume reduction in the planned cold or hot spots, as well as the shift in location of the cold/ hot voxels. (Fig. 5, B).

\subsubsection{Directional gradient metrics}

The simulated directional gradient dose distributions were sensitive to simulated setup errors in regions with steep dose gradients. This can be seen by the spread in values of the $\mathrm{GI}_{\mathrm{dir}}, 3 \mathrm{~cm}$. Fig. 5 panel $\mathrm{C}$ shows values for the scenario with a shallower gradient in the anterior direction (see Fig. 1, A). The dose change at $3 \mathrm{~cm}$ in the anterior direction, where the gradient is less steep, was very robust towards simulated setup errors. The dose change in the left direction, where the gradient is steep, varied under the influence of setup errors. The mean gradient index GCI was overall more robust towards the effects of setup errors than the

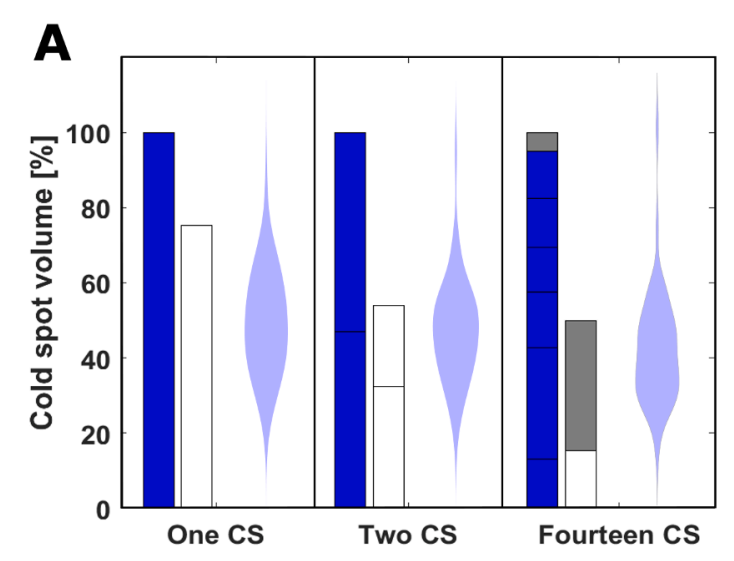

B
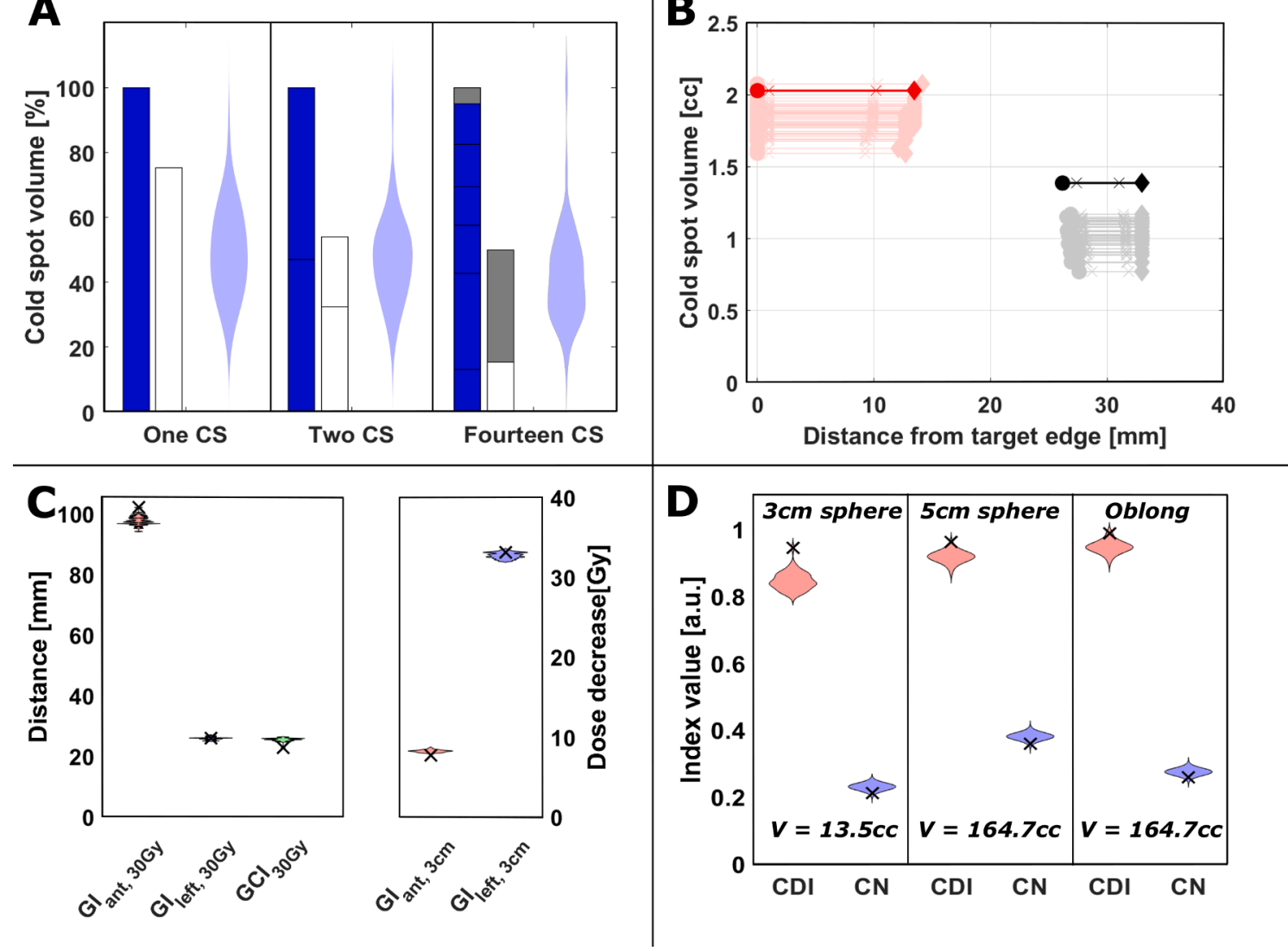

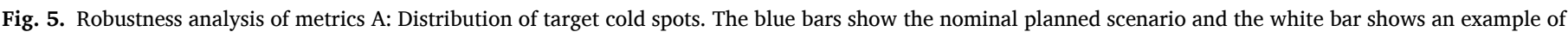

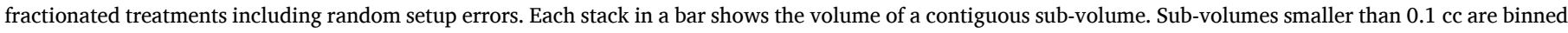

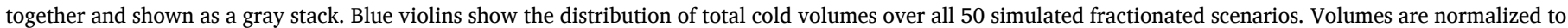

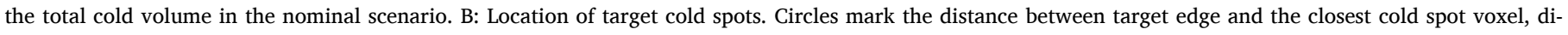

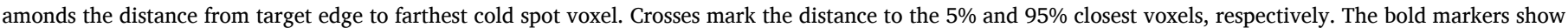

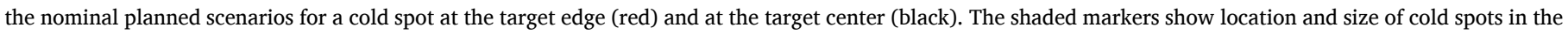

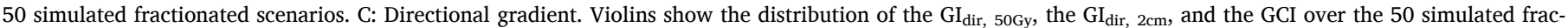

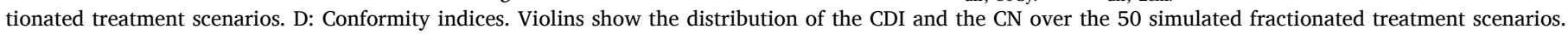

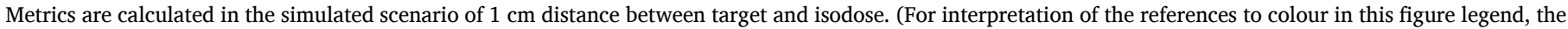
reader is referred to the web version of this article.) 
directional indices if evaluated at higher dose levels ( $40 \mathrm{~Gy}$ and above in this analysis). When evaluated at lower dose levels (30 Gy and below, corresponding to $50 \%$ target dose, in this analysis) the GCI tended towards higher values (i.e. showing a shallower gradient) in fractionated scenarios (see Supplementary Figure S8.

\subsubsection{Conformity, gradient, and homogeneity metrics}

The dose blurring in fractionated scenarios made the simulated isodose volumes slightly smaller, which was reflected in the values of mean conformity and mean gradient indices (Fig. 5, D). All metrics retained their behavior with varying target volume and shape: those that were dependent on shape and volume remained so, as did those that were not.

The target's differential DVH was skewed towards lower doses in fractionated scenarios. This increased heterogeneity was only reflected in the value of $\mathrm{HI}_{\mathrm{ICRU}}$. The remaining HIs showed values suggesting an increased homogeneity in fractionated scenarios, due to lower absolute maximum target doses.

\subsection{Our suggestion for a metrics toolbox}

We selected metrics to include in our toolbox based on the following criteria. First, the metrics should be able to describe the simulated dose distribution changes. They should also, as far as possible, be independent of the shape and size of the target (or OAR) in the given patient anatomy, and this independence should ideally be preserved in the presence of random setup errors. Finally, it should be possible to explain the metrics and their utility to clinical users without excessive questioning. In the case of GIs, calculation simplicity was another factor. Since our favored metric, the GCI, can be quite calculation-intensive depending on the available programming interface, we included the simpler $\mathrm{GI}_{\text {dist }}$ alongside it. Analogously, in the case of CIs we included two complementary metrics: one that could describe all simulated scenarios (and was simple to calculate) and one that was independent of target shape and size (and was potentially computation-intensive).

Based on the results described in the previous section, we identified the following metrics as the most useful additional tools for quantitative plan quality assessment. These constitute a set of indices giving comprehensive and complementary information on the three-dimensional dose distribution that is not contained in standard DVH metrics.

\section{Conformity:}

- CDI - Conformity Distance Index [13] as defined in Table 1

- CN - Conformity Number [7] as defined in Table 1

\section{Homogeneity:}

- $\mathrm{HI}_{\mathrm{ICRU}}$ - Homogeneity Index proposed in ICRU report 83 [14] as defined in Table 1

\section{Gradient (overall):}

- GCI - Gradient Curve Index [22] as defined in Table 1

- $\mathrm{GI}_{\text {dist }}$ - effective distance between two isodose volumes $[9,20,31]$ as defined in Table 1

\section{Gradient (directional):}

- $\mathrm{GI}_{\mathrm{dir}, \mathrm{x}, \mathrm{d}}$ and $\mathrm{GI}_{\mathrm{dir}, \mathrm{x}, \mathrm{D}}$ - directional Gradient Index variants as introduced in section 2.1.2

- $\mathrm{GI}_{\mathrm{OAR}}$ - directional Gradient Index as introduced in section 2.1.2

\section{Distribution of cold/hot spots:}

- Size and volume of contiguous cold/hot spots

\section{Location of cold/hot spots:}

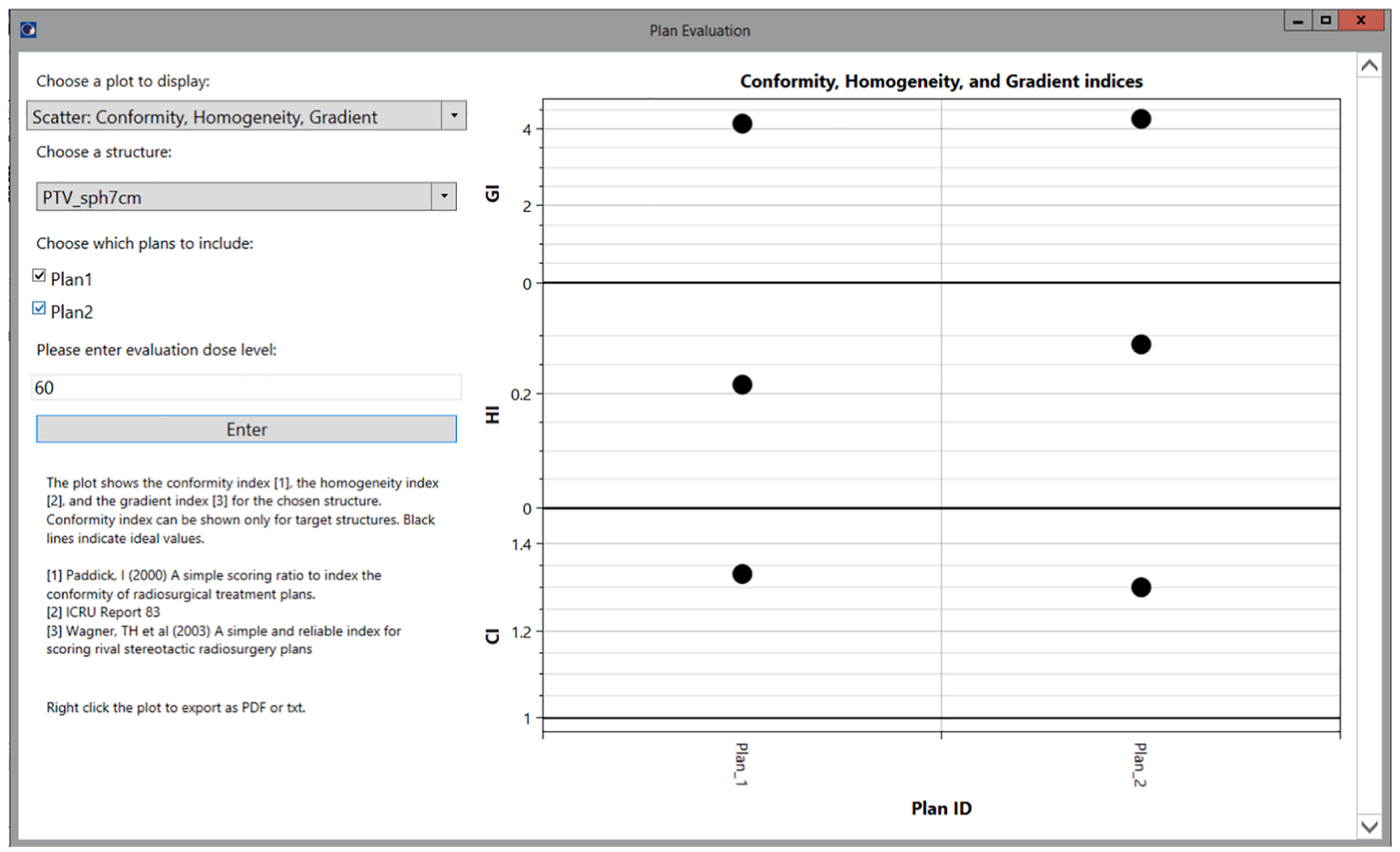

Fig. 6. Screenshot of the PlanEvaluation user interface as it is run in Eclipse v. 13.7. The plot shows GI, HI, and CI for the two plans shown in Fig. 2 panels F ("Plan_1", left) and G ("Plan_2", right). 
- VDist $_{\min / \max }$ as introduced in section 2.1.3

We implemented calculation and visualization of these metrics in the PlanEvaluation ESAPI plugin for the Eclipse TPS (a link to the software repository is given at the end of this paper).

\subsection{The PlanEvaluation plugin script}

Figure 6 shows a screenshot of the implemented ESAPI script as it is run in Eclipse v 13.7. A treatment planner can run the script from within the Eclipse user interface once the dose has been calculated for one or several plans they wish to evaluate and compare. Upon running the script, a separate window with the interface shown in Fig. 6 appears. The desired metric and the structure for which it should be calculated can be chosen from drop-down menus, after which the planner selects the plan (s) they wish to evaluate. If the metric requires additional input by the user, corresponding input boxes are shown upon selection of the metric. When a plan is selected from the list, the chosen metric is visualized in the plot on the right for this plan. A metric can be shown for several plans at once in order to compare them. Plots and underlying data can be exported in PDF, csv, or txt format, respectively. Most metrics can also be calculated and visualized for previously calculated uncertainty scenarios, based on the Eclipse version used.

\section{Discussion}

In this work, we give a systematic evaluation and comparison of metrics that quantify clinically interesting aspects of the spatial dose distribution. We have selected a set of optimal, complimentary metrics for comprehensive evaluation of the spatial dose. In addition, we have implemented these metrics in a ready-to-use toolbox to aid in treatment planning. Even though our implementation is specific to the Eclipse treatment planning system, the methodology can easily be generalized as most treatment planning systems today offer advanced scripting access.

The robustness analysis of the metrics showed that fractionation effects are an important factor to consider when evaluating plan quality metrics in the planned static dose distribution. Some metrics, such as mean gradient and conformity indices, showed only minimal variation between the nominal and fractionated scenarios. Others, such as volume and size of cold spots, varied notably between nominal and fractionated scenarios - and between the individual simulated courses. An interesting observation was that the GCI tended towards showing shallower gradient values for lower evaluation isodoses, even though the isodose volumes were smaller in fractionated scenarios than in the nominal dose. This is because the lower isodose volumes had more irregular shapes in the simulated treatment plans. In the fractionated scenarios, the isodoses were blurred into more regular shapes with smaller surface areas (see Supplementary Figure S8. If well characterized, the observed metric behaviors could be useful in predicting the effect of positioning errors on the spatial dose property in question.

Commercial solutions for incorporating some quantitative spatial dose information in the treatment plan evaluation process have recently been introduced, most prominently SunNuclear's PlanIQ ${ }^{\mathrm{TM}}$ (SunNuclear Corporation, Melbourne, USA) and the ProKnow plan evaluation software recently incorporated into Elekta's treatment planning solution (Elekta AB, Stockholm, Sweden). Users are constrained to the metrics provided by the vendor, however, and to our knowledge, these are still limited to DVH-based conformity, homogeneity, and gradient indices.

Metrics to quantify dose conformity, homogeneity, and gradient have been collected and reviewed in several previous publications $[5,14,19,26,46-48]$. Most of these evaluate different indices in a specific clinical context, e.g. stereotactic treatment of small brain tumors. The ICRU gives general recommendations on conformity and homogeneity metrics to use in photon treatment planning, as well as a gradient metric for stereotactic treatments $[14,26]$. The ICRU reports published to date do not provide any suggestions for metrics to quantify the dose distribution in more detail, however.

In the absence of quantitative measures for plan quality, the evaluation of treatment plans is a subjective process and can thus be subject to variations between individual evaluators. Minimizing subjective variations in treatment planning and plan quality evaluation can lead to an overall global increase in plan quality. In radiotherapy treatment planning, standardization requires two steps: consensus on which parameters constitute a "good" plan in the given treatment setting, and metrics to consistently quantify how a plan performs on these parameters. The first is a clinical consideration specific to the treatment type, and often the individual patient. The second is the issue we seek to address with this work.

The main aim of this study was the systematic, quantitative comparison of the various metrics and based on this the selection of an optimal set. A secondary aim was to demonstrate the potential of providing a metrics tool to help in daily clinical practice, hence intuitive and easy visualization was included as a selection criterion. This selection of visualization methods is, by its nature, based on more qualitative assessments in collaboration with practicing clinicians. Some degree of subjectivity and personal bias in this selection process could not be avoided - especially since all clinicians were from the same institution. Nevertheless, we believe the suggestions we present here will provide a good starting point for clinics who wish to implement more quantitative metrics in their clinical plan comparison routine.

It is important to stress that the aim of this study is not to determine what constitutes a "best" treatment plan. This question is specific to the type of disease, anatomical site and, often, each individual patient. For example, a steep dose gradient towards the spinal cord might be undesirable if plan robustness is a concern, while stereotactic treatments rely on sharp gradients. Instead, we present a comprehensive toolbox from which various metrics that may be deemed appropriate for a specific case can be chosen and easily evaluated. We are currently investigating this using clinical examples, both retrospectively and prospectively in clinical treatment planning situations.

Further than aiding in clinical plan evaluation, it is possible that these metrics could be incorporated into the plan optimization process itself. This could be feasible by either directly incorporating metrics as cost functions or by finding adequate convex surrogate functions for faster optimization and avoidance of local minima. This could also enable the exploration of trade-offs between a spatial dose metric and various DVH parameters in a multi-criteria optimization setting.

We selected the metrics to include in the proposed toolbox based on simulated scenarios of extreme variations in the spatial dose distribution. Such extreme variations are not likely to occur in clinical dose distributions, and one could thus argue against using these unrealistic scenarios to test the performance of plan quality metrics. Exaggerating the size of possible dose variations makes the differences between individual metrics clearer, however, and thus makes it easier to identify whether a metric reacts as desired to specific changes. The clinical utility of the selected metrics has continuously been discussed with experienced clinical medical physicists and radiation oncologists.

Apart from clinical plan evaluation, another area in which consistency in treatment plan comparison is important is that of reporting in audit and research studies $[40,49]$. Quantitative metrics are essential to accurately evaluate audit plans and compare competing plans in insilico planning studies, as well as in clinical studies in which reported toxicities must be correlated with well-defined quality metrics of the delivered dose. Some of the metrics discarded for the purpose of daily clinical plan evaluation may be useful in other contexts. For example, dosiomics metrics such as the $\operatorname{LCS}_{\mathrm{D}}$ have been shown to add value to predictive outcome models [33-38,50,51].

In this work we have focused solely on metrics to quantify spatial properties of the simulated dose distribution. Overall treatment plan quality comprises many other aspects, including for example robustness, complexity, and delineation accuracy $[40,41]$. The question of specific 
metrics aimed at quantifying robustness becomes especially interesting when comparing photon and proton dose distributions. Quantitative metrics are undoubtedly equally useful and important in all of these other contexts but covering this was outside of the scope of our present work.

The ESAPI PlanEvaluation scripts and the script used for metrics comparison (including DICOM files of phantom, structures, and simulated plans) can be seen and downloaded freely at https://gitlab. com/LPKaplan/esapi-quantitative-plan-comparison/-/tree/master. We appreciate and encourage anyone with an interest in treatment plan quality assessment to contact us for feedback, discussion, or possible future collaboration.

In conclusion, we present a ready-to-use toolbox of rigorously selected metrics to quantify clinically relevant spatial aspects of a dose distribution. This work serves as a step towards a broader consensus on the use of spatial dose metrics in radiotherapy.

\section{Funding}

This work was supported by DCCC Radiotherapy - The Danish National Research Center for Radiotherapy, Danish Cancer Society (grant no. R191-A11526) and Danish Comprehensive Cancer Center, and the Danish Cancer Society (grant no R167-A11003).

\section{Declaration of Competing Interest}

The authors declare that they have no known competing financial interests or personal relationships that could have appeared to influence the work reported in this paper.

\section{Acknowledgements}

We kindly thank Anne Ivalu Sander Holm, Anne Vestergaard, Lone Hoffmann, Ditte Sloth Møller, Ulrik Vindelev Elstrøm, Jesper Grau Eriksen, and Kenneth Jensen for providing clinical insights and input, Jasper Nijkamp and Katarína Furmanová for helping with visualization ideas, and Kari Tanderup, Jasper Nijkamp and Esben Schjødt Worm for reviewing and discussing the manuscript.

\section{Appendix A. Supplementary data}

Supplementary data to this article can be found online at https://doi. org/10.1016/j.ejmp.2021.09.014.

\section{References}

[1] Marks LB, Yorke ED, Jackson A, Ten Haken RK, Constine LS, Eisbruch A, et al. Use of normal tissue complication probability models in the clinic. Int $\mathrm{J}$ Radiat Oncol Biol Phys 2010;76(3):S10-9. https://doi.org/10.1016/j.ijrobp.2009.07.1754.

[2] Kaplan LP, Holm AIS, Elstrøm UV, Eriksen JG, Jensen K, Primdahl H, et al. OC0520 Inter-observer variations in plan evaluation. Radiother Oncol 2019;133: S271-2. https://doi.org/10.1016/S0167-8140(19)30940-5.

[3] Petersson K, Engellau J, Nilsson P, Engström P, Knöös T, Ceberg C. Treatment plan comparison using grading analysis based on clinical judgment. Acta Oncol 2013;52 (3):645-51. https://doi.org/10.3109/0284186X.2012.734926.

[4] Njeh CF, Parker BC, Orton CG. Evaluation of treatment plans using target and normal tissue DVHs is no longer appropriate: point/Counterpoint. Med Phys 2015; 42(5):2099-102. https://doi.org/10.1118/1.4903902.

[5] Feuvret L, Noël G, Mazeron J-J, Bey P. Conformity index: a review. Int J Radiat Oncol Biol Phys 2006;64(2):333-42. https://doi.org/10.1016/j. ijrobp.2005.09.028.

[6] Paddick I. A simple scoring ratio to index the conformity of radiosurgical treatment plans. J Neurosurg 2000;93:219-22.

[7] Riet AV, Mak ACA, Moerland MA, Elders LH, van der Zee W. A conformation number to quantify the degree of conformality in brachytherapy and external beam irradiation: application to the prostate. Int J Radiat Oncol Biol Phys 1997;37(3): $731-6$.

[8] Baltas D, Kolotas C, Geramani K, Mould RF, Ioannidis G, Kekchidi M, et al. A conformal index (COIN) to evaluate implant quality and dose specification in brachytherapy. Int J Radiat Oncol Biol Phys 1998;40(2):515-24.

[9] Leung LHT, Chua DTT, Wu PM. A new tool for dose conformity evaluation of radiosurgery treatment plans. Int J Radiat Oncol Biol Phys 1999;45(1):233-41.
[10] Lomax NJ, Scheib SG. Quantifying the degree of conformity in radiosurgery treatment planning. Int J Radiat Oncol Biol Phys 2003;55(5):1409-19. https://doi. org/10.1016/S0360-3016(02)04599-6.

[11] Shaw E, Kline R, Gillin M, Souhami L, Hirschfeld A, Dinapoli R, et al. Radiation Therapy Oncology Group: radiosurgery quality assurance guidelines. Int J Radiat Oncol Biol Phys 1993;27(5):1231-9.

[12] Park JM, Park S-Y, Ye S-J, Kim JH, Carlson J, Wu H-G. New conformity indices based on the calculation of distances between the target volume and the volume of reference isodose. BJR 2014;87(1043):20140342. https://doi.org/10.1259/ bjr.20140342.

[13] Wu Q-R, Wessels BW, Einstein DB, Maciunas RJ, Kim EY, Kinsella TJ. Quality of coverage: conformity measures for stereotactic radiosurgery. J Appl Clin Med Phys 2003;4(4):374-81.

[14] ICRU Report 83: Prescribing, Recording, and Reporting Photon-Beam IntensityModulated Radiation Therapy (IMRT). Journal of the ICRU 2010;10:NP.3-NP. 10.1093/jicru/ndq002.

[15] Yoon M, Park SY, Shin D, Lee SB, Pyo HR, Kim DY, et al. A new homogeneity index based on statistical analysis of the dose-volume histogram. J Appl Clin Med Phys 2007:8(2):9-17.

[16] Heufelder J, Zink K, Scholz M, Kramer K-D, Welker K. Eine Methode zur automatischen Bewertung von CT-basierten Bestrahlungsplänen in der perkutanen Strahlentherapie. Z Med Phys 2003;13:231-9.

[17] Miften MM, Das SK, Su M, Marks LB. A dose-volume-based tool for evaluating and ranking IMRT treatment plans. J Appl Clin Med Phys 2004;5(4):1-14.

[18] Wu Q, Mohan R, Morris M, Lauve A, Schmidt-Ullrich R. Simultaneous integrated boost intensity-modulated radiotherapy for locally advanced head-and-neck squamous cell carcinomas. I: dosimetric results. Int J Radiat Oncol Biol Phys 2003; 56(2):573-85.

[19] Ohtakara K, Hayashi S, Hoshi H. Dose gradient analyses in Linac-based intracranial stereotactic radiosurgery using Paddick's gradient index: consideration of the optimal method for plan evaluation. JRR 2011;52(5):592-9. https://doi.org/ 10.1269/jrr.11005.

[20] Wagner TH, Bova FJ, Friedman WA, Buatti JM, Bouchet LG, Meeks SL. A simple and reliable index for scoring rival stereotactic radiosurgery plans. Int J Radiat Oncol Biol Phys 2003;57(4):1141-9.

[21] Paddick I, Lippitz B. A simple dose gradient measurement tool to complement the conformity index. J Neurosurg 2006;105(Supplement):194-201.

[22] Sung K, Choi YE, Zhang Q. Dose gradient curve: a new tool for evaluating dose gradient. PLoS One 2018;13(4):e0196664. https://doi.org/10.1371/journal. pone.0196664.

[23] Mayo CS, Ding L, Addesa A, Kadish S, Fitzgerald TJ, Moser R. Initial experience with volumetric IMRT (RapidArc) for intracranial stereotactic radiosurgery. Int $\mathrm{J}$ Radiat Oncol Biol Phys 2010;78(5):1457-66. https://doi.org/10.1016/j. ijrobp.2009.10.005.

[24] Wang X, Zhang X, Dong L, Liu H, Gillin M, Ahamad A, et al. Effectiveness of noncoplanar IMRT planning using a parallelized multiresolution beam angle optimization method for paranasal sinus carcinoma. Int J Radiat Oncol Biol Phys 2005;63(2):594-601.

[25] Dimitriadis A, Paddick I. A novel index for assessing treatment plan quality in stereotactic radiosurgery. J Neurosurg 2018;129:118-24. https://doi.org/ 10.3171/2018.7.GKS18694.

[26] Report ICRU. 91: Prescribing, recording, and reporting of stereotactic treatments with small photon beams. J ICRU 2014;14:101-9. https://doi.org/10.1093/jicru/ ndx010.

[27] Lefkopoulos D, Dejean C, El-Balaa H, Platoni K, Grandjean P, Foulquier J-N, et al. In: The Use of Computers in Radiation Therapy. Berlin, Heidelberg: Springer Berlin Heidelberg; 2000. p. 356-8. https://doi.org/10.1007/978-3-642-59758-9_135.

[28] Zhao Bo, Joiner MC, Orton CG, Burmeister J. "SABER": A new software tool for radiotherapy treatment plan evaluation: Spatial and biological radiotherapy plan evaluation. Med Phys 2010;37(11):5586-92. https://doi.org/10.1118/1.3497152.

[29] Said M, Nilsson P, Ceberg C. Analysis of dose heterogeneity using a subvolumeDVH. Phys Med Biol 2017;62:N517-24. https://doi.org/10.1088/1361-6560/ aa8b0a.

[30] Deasy JO, Blanco AI, Clark VH. CERR: a computational environment for radiotherapy research. Med Phys 2003;30(5):979-85. https://doi.org/10.1118/ 1.1568978

[31] Mayo CS, Zankowski C, Herman M, Miller R, Olivier K, Vincent A, et al. A method to vectorize the dose distribution, the dose volume histogram and create a dose vector histogram: VDD, VDVH. DVctH. Med Phys 2013;40(1):011717. https://doi. org/10.1118/1.4769111.

[32] Cheung FWK, Law MYY. A novel conformity index for intensity modulated radiation therapy plan evaluation: conformity index for intensity modulated radiation therapy plan. Med Phys 2012;39(9):5740-56. https://doi.org/10.1118/ 1.4742848 .

[33] Rossi L, Bijman R, Schillemans W, Aluwini S, Cavedon C, Witte M, et al. Texture analysis of $3 \mathrm{D}$ dose distributions for predictive modelling of toxicity rates in radiotherapy. Radiother Oncol 2018;129(3):548-53. https://doi.org/10.1016/j. radonc.2018.07.027.

[34] Gabryś HS, Buettner F, Sterzing F, Hauswald H, Bangert M. Design and selection of machine learning methods using radiomics and dosiomics for normal tissue complication probability modeling of xerostomia. Front Oncol 2018;8:35. https:// doi.org/10.3389/fonc.2018.00035.

[35] Wu A, Li Y, Qi M, Lu X, Jia Q, Guo F, et al. Dosiomics improves prediction of locoregional recurrence for intensity modulated radiotherapy treated head and neck cancer cases. Oral Oncol 2020;104:104625. https://doi.org/10.1016/j. oraloncology.2020.104625. 
[36] Liang B, Yan H, Tian Y, Chen X, Yan L, Zhang T, et al. Dosiomics: extracting 3D spatial features from dose distribution to predict incidence of radiation pneumonitis. Front Oncol 2019;9. https://doi.org/10.3389/fonc.2019.00269.

[37] Buettner F, Gulliford SL, Webb S, Sydes MR, Dearnaley DP, Partridge M. Assessing correlations between the spatial distribution of the dose to the rectal wall and late rectal toxicity after prostate radiotherapy: an analysis of data from the MRC RT01 trial (ISRCTN 47772397). Phys Med Biol 2009;54(21):6535-48. https://doi.org/ 10.1088/0031-9155/54/21/006.

[38] Dean J, Wong K, Gay H, Welsh L, Jones A-B, Schick U, et al. Incorporating spatial dose metrics in machine learning-based normal tissue complication probability (NTCP) models of severe acute dysphagia resulting from head and neck radiotherapy. Clin Transl Radiat Oncol 2018;8:27-39. https://doi.org/10.1016/j. ctro.2017.11.009.

[39] Placidi L, Lenkowicz J, Cusumano D, Boldrini L, Dinapoli N, Valentini V. Stability of dosomics features extraction on grid resolution and algorithm for radiotherapy dose calculation. Phys Med 2020;77:30-5. https://doi.org/10.1016/j. ejmp.2020.07.022.

[40] Hernandez V, Hansen CR, Widesott L, Bäck A, Canters R, Fusella M, et al. What is plan quality in radiotherapy? The importance of evaluating dose metrics, complexity, and robustness of treatment plans. Radiother Oncol 2020;153:26-33. https://doi.org/10.1016/j.radonc.2020.09.038.

[41] Moore KL, Brame RS, Low DA, Mutic S. Quantitative metrics for assessing plan quality. Semin Radiat Oncol 2012;22(1):62-9. https://doi.org/10.1016/j. semradonc.2011.09.005.

[42] Rosenfeld A, Pfaltz JL. Sequential operations in digital picture processing. JACM 1966;13(4):471-94.
[43] Dasarathy BV, Holder EB. Image characterizations based on joint gray level—run length distributions. Pattern Recogn Lett 1991;12(8):497-502.

[44] Bentley JL. Multidimensional binary search trees used for associative searching. Commun ACM 1975;18(9):509-17. https://doi.org/10.1145/361002.361007.

[45] Dice LR. Measures of the Amount of Ecologic Association Between Species. Ecology 1945;26:297-302. https://doi.org/10.2307/1932409.

[46] Kataria T, Sharma K, Subramani V, Karrthick KP, Bisht S. Homogeneity index: an objective tool for assessment of conformal radiation treatments. J Med Phys 2012; 37(4):207. https://doi.org/10.4103/0971-6203.103606.

[47] Yaparpalvi R, Garg MK, Shen J, Bodner WR, Mynampati DK, Gafar A, et al. Evaluating which plan quality metrics are appropriate for use in lung SBRT. BJR 2018:20170393. https://doi.org/10.1259/bjr.20170393.

[48] Patel G, Mandal A, Choudhary S, Mishra R, Shende R. Plan evaluation indices: a journey of evolution. Rep Pract Oncol Radiother 2020;25(3):336-44. https://doi. org/10.1016/j.rpor.2020.03.002.

[49] Hansen CR, Crijns W, Hussein M, Rossi L, Gallego P, Verbakel W, et al. Radiotherapy Treatment plannINg study Guidelines (RATING): a framework for setting up and reporting on scientific treatment planning studies. Radiother Oncol 2020;153:67-78. https://doi.org/10.1016/j.radonc.2020.09.033.

[50] Lee R, Chan EK, Kosztyla R, Liu M, Moiseenko V. Dose-distance metric that predicts late rectal bleeding in patients receiving radical prostate external-beam radiotherapy. Phys Med Biol 2012;57(24):8297-307. https://doi.org/10.1088/ 0031-9155/57/24/8297.

[51] Buettner F, Gulliford SL, Webb S, Partridge M. Modeling late rectal toxicities based on a parameterized representation of the 3D dose distribution. Phys Med Biol 2011; 56(7):2103-18. https://doi.org/10.1088/0031-9155/56/7/013. 